\title{
Constructive Interference in Steady State Imaging in the Central Nervous System
}

Fabrício Guimarães Gonçalves ${ }^{1}$ and Lázaro Luis Faria do Amaral²

1. Neuroradiology Fellow, Montreal General Hospital, McGill University Health Center;

2. Neuroradiologist, and Chief, Neuroradiology Department, Hospital da Real Beneficiência Portuguesa de São Paulo

\begin{abstract}
Constructive interference in steady state (CISS) is a fully refocused fast-gradient echo sequence that is mainly used in the assessment of the central nervous system. The most important advantages of steady-state imaging are short acquisition times, high signal-to-noise ratio, and better contrast-to-noise ratio. Owing to its cisternographic effect, CISS is useful in the assessment of the cranial nerves, and can also be used when studying cysts, cystic masses, and neurocysticercosis and in hydrocephalus cases. CISS has been shown to be useful in spinal imaging, epecially in cases of arteriovenous malformation and when it is helpful to better characterise intra- and extramedullary cystic abnormalities.
\end{abstract}

\section{Keywords}

Magnetic resonance imaging, fast-gradient echo sequence, steady-state imaging, constructive interference in steady state, longitudinal magnetisation, transverse magnetisation, central nervous system, cranial nerves, neurocysticercosis, spine

Disclosure: The authors have no conflicts of interest to declare.

Acknowledgements: The authors would like to make a special acknowledgment to Dr Michael McInnis for reviewing the material and for useful suggestions.

Received: 8 July 2010 Accepted: 11 October 2010 Citation: European Neurological Review, 2011;6(2):138-142 DOI:10.17925/ENR.2011.06.02.138

Correspondence: Fabrício Guimarães Gonçalves, Diagnostic Radiology, Montreal General Hospital, 1650 Cedar Avenue, Room D5 137, Montreal, Quebec, Canada, H3G 1A4. E: goncalves.neuroradio@gmail.com

\section{What Is Constructive Interference in Steady State Imaging?}

Constructive interference in steady state (CISS) imaging is a member of the family of fast gradient echo (GRE) sequences. The CISS sequence is particularly dependent on high gradient amplitude and slew rates.' To run this type of sequence, high-gradient-strength magnets are required to reach the peak of the gradient as rapidly as possible. CISS has different names according to different manufacturers: it is called fast imaging employing steady-state acquisition (FIESTA) by General Electric, true fast imaging with steady-state precession (FISP) by Siemens, balanced fast field echo (FFE) by Philips, and true steady-state free precession (SSFP) by Toshiba. CISS is mainly used in the assessment of the central nervous system, but it is also used when imaging the abdomen, the musculoskeletal system and the breast. The last three areas are not within the scope of this article.

\section{How Are Constructive Interference in Steady State Images Acquired?}

When inside a magnetic resonance imaging (MRI) scanner, the randomly moving protons in the patient's body align along the longitudinal z-axis The z-axis is a magnetisation vector parallel to both the long axis of the patient's body and the bore of the scanner. This magnetisation force, represented as a vector along the positive side of the z-axis, is called longitudinal magnetisation (LM). When a radiofrequency pulse (RFP) is applied, the magnetisation vector is tipped to the transverse plane. This tipped magnetisation vector has two components: the LM, which recovers, and the transverse magnetisation (TM), which decays during each repetition time (TR) period in the $x$-axis.

CISS is a 'green' sequence. The main recycling idea is not to waste the signal from the decaying TM in every RFP, because it is this signal that provides the T2 properties of a given tissue. Instead of being lost, the TM is reinforced into the transverse plane. The TM is recovered by applying an $180^{\circ}$ phase shift during each TR period, after which the TM shifts in the transverse plane. The following RFP simultaneously tips a component of the residual TM back along the z-axis and a portion of the LM into the transverse plane (X-axis). After multiple TR periods have elapsed, this feeding of the LM into the TM, and vice versa, eventually establishes an equilibrium state of both the LM and the TM.

It must be stressed that it is necessary to keep the TR shorter than the T2 relaxation time of the tissue; in this way there is insufficient time for the TM to decay completely before the next RFP excitation. To achieve CISS images, residual TM is always necessary. Once the equilibrium is reached, two types of signal are produced: the first is a post-excitation signal, which consists of free induction decay arising from the most recent RFP, and the second is the echo reformation prior to excitation, when residual echo is refocused at the time of the subsequent RFP.

There are three main types of fast GRE sequence: spoiled, partially refocused and fully refocused. When the pre- and post-excitation signals are lost and wasted, the resulting image is T1-weighted. When only the post-excitation signal is acquired, the images obtained are 
very sensitive to magnetic field inhomogeneities, with a resulting T2* (star) effect. A very similar image to CISS can be obtained by acquiring the pre-excitation signal only. This sequence has a relatively long echo time (TE) and therefore suffers from more flow artifacts and a lower signal-to-noise ratio. CISS is a fully refocused fast GRE sequence in which both pre- and post-excitation signals are sampled, with the subsequent advantage of having a high signal-to-noise ratio and high spatial resolution.

\section{Advantages of Constructive Interference in Steady State Imaging}

One of the most important advantages of steady-state imaging is the short acquisition time thanks to the short TR and TE. With short TR and $\mathrm{TE}$, tissues with long $\mathrm{T} 2$ relaxation will demonstrate wider range and additional signals owing to various refocused echo paths. Other advantages are the high signal-to-noise ratio and better contrast-to-noise ratio. Additionally, CISS images do not present significant susceptibility-, motion-, or flow-related artifacts. ${ }^{2}$

\section{Uses of Constructive Interference in Steady State}

CISS is widely used to assess the cranial nerves (CNS). The CNs were first studied by CISS at the beginning of the 1990s, and the first CNS studied were the facial (CNVII) and vestibulocochlear (CNVIII) nerves. ${ }^{3}$ Owing to its cisternographic effect, CISS provides finely detailed images of the CNS, especially of their cisternal and canalicular portions (see Figures 1 and 2).

In cases of pulsatile tinnitus or facial spasms, CISS plays a major role in the assessment of vascular loops compressing the nerves in the cerebellopontine angle cistern (CPA) or inside the internal auditory canal (IAC) (see Figure 3). ${ }^{4}$ This sequence is also useful to study masses in the CPA cistern, especially in cases of hearing impairment. ${ }^{5}$

\section{The Cranial Nerves}

The Olfactory and Optic Nerves

CISS can be used in the evaluation of the olfactory nerve (CNI). It provides better results than 2D turbo spin echo, although not significantly better in comparison with ultrafast spoiled gradient echo 3D imaging (multiplanar gradient-recalled [MPGR]/magnetisationprepared rapid acquisition gradiant echo [MPRAGE]). ${ }^{6}$ CISS provides similar results to MPRAGE in the assessment of the optic nerve (CNII), although MPRAGE is superior to CISS for studying the optic chiasm and tract. $^{\text {? }}$

\section{The Oculomotor Nerve}

The oculomotor nerve (CNIII) is readily seen from the interpeduncular fossa to the cavernous sinuses (see Figure 4). ${ }^{8}$ The CNIII runs between the posterior cerebral artery (PCA) and the superior cerebellar artery (SCA) and innervates the extraocular muscles, including the elevator muscle of the upper eyelid, except for the superior oblique and the lateral rectus muscles. CISS can potentially be used to rule out aneurysms arising from the posterior circulation or from the posterior communicating artery that impinge on the CNIII. ${ }^{9}$

\section{The Trochlear Nerve}

The trochlear nerve (CNIV) is the longest intracranial nerve, although it is the smallest in terms of number of axons. It originates in the dorsal aspect of the midbrain and innervates the superior oblique muscle. It runs posteriorly and inferiorly around the Sylvian aqueduct
Figure 1: Axial Constructive Interference in Steady State Image of the Posterior Fossa at the Level of the Inner Ear Demonstrating the Normal Anatomy of the Cisternal and Canalicular Portions of the Seventh and Eighth Nerves

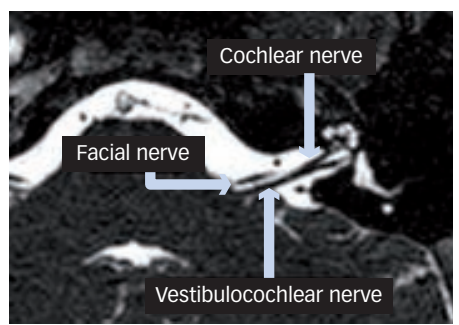

Figure 2: Sagittal Constructive Interference in Steady State Image Through the Internal Auditory Canal, Showing the Facial, Cochlear and the Superior and Inferior Vestibular Nerves

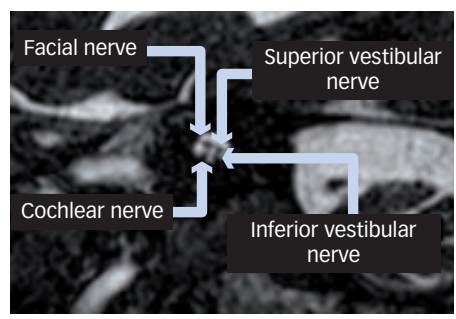

Figure 3: Axial Constructive Interference in Steady State Images of the Posterior Fossa, Centered at the Right Inner Ear, in a Patient with Pulsatile Tinnitus

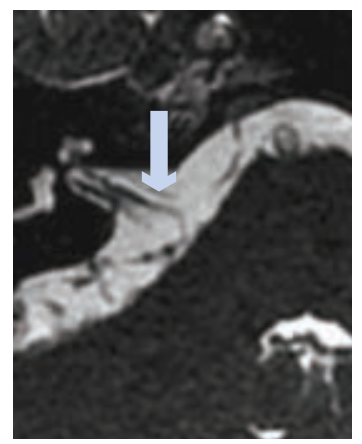

There is an anterior inferior cerebellar artery loop inside the right internal auditory canal (arrow) in close association with and likely compressing the seventh and eighth nerves.

Figure 4: Axial Constructive Interference in Steady State Image at Level of Interpeduncular Fossa Demonstrating the Cisternal Portions of the Oculomotor Nerves

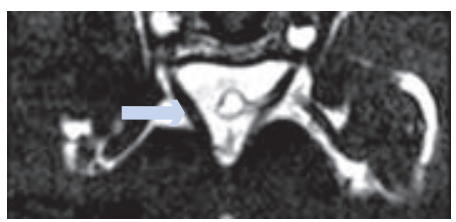

Arrow showing the right oculomotor nerve (CNIII).

and decussates at the level of the superior medullary velum. CNIV exists beneath the inferior colliculus, opposite its nucleus of origin. The cisternal segment of the CNIV parallels the courses of the SCA, the PCA, and the basal vein of Rosenthal, passing between the PCA and the SCA, piercing the dura and reaching the cavernous sinus. CISS 
Figure 5: Sagittal Oblique Reformatted Constructive Interference in Steady State Image Centred at the Level of the Prepontine Cistern

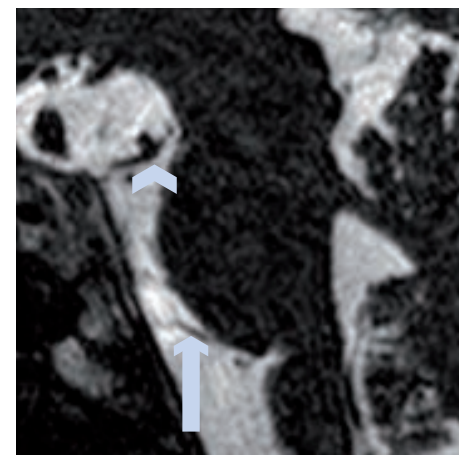

The normal cisternal portion of the abducens nerve (arrow) is seen. The oculomotor nerve (arrowhead) is seen between the posterior cerebral artery (top) and the superior cerebellar artery (bottom).

Figure 6: Axial Constructive Interference in Steady State Image at the Level of the Facial Coliculli (Small Arrows) Demonstrating both Trigeminal Nerves in their Cisternal Portions

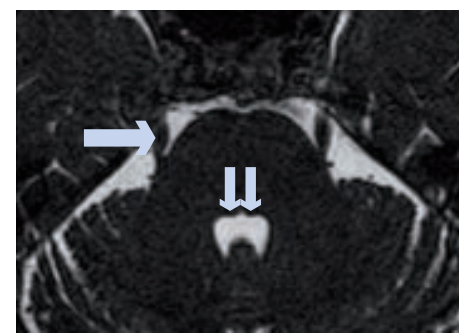

Note the normal anatomy of the right trigeminal nerve (CNV; large arrow).

Figure 7: Axial Constructive Interference in Steady State Image at the Level of the Jugular Foramen Showing the Glossopharyngeal Nerve (Large Arrow) Entering the Pars Nervosa of the Jugular Foramen in the Left Side (Small Arrow)

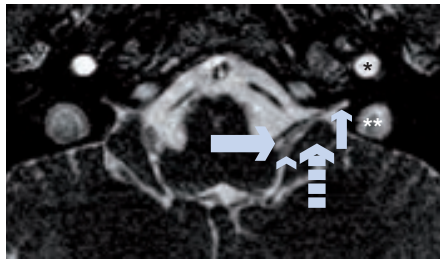

The glossopharyngeal nerve is seen anterior to the choroid plexus (arrow head) and to the flocullus cerebelli (dashed arrow).

*Internal carotid artery **Jugular bulb.

Figure 8: Axial Constructive Interference in Steady State Image at the Level of the Inferior Olivary Nucleus Showing the Normal Left Vagus Nerve

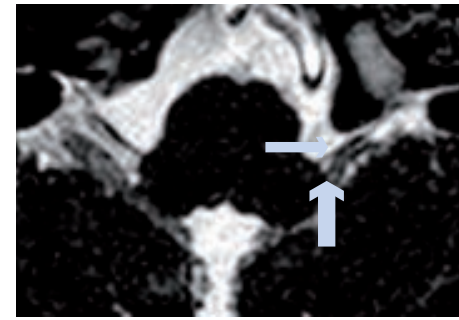

The two rootlets of the vagus nerve (large arrow) are seen posteriorly to the glossopharyngeal nerve (small arrow) entering the pars vascularis of the jugular foramen. allows accurate identification of the cisternal segment of the CNIV and its neurovascular relationships in the vast majority of cases. ${ }^{10}$

The Trigeminal Nerve

The trigeminal nerve (CNV) is the largest $\mathrm{CN}$ in terms of number of axons. It is mainly a sensory nerve of the face and has three main divisions. The first (V1) and second (V2) divisions (ophthalmic and maxillary) are purely sensory, while the third (V3) (mandibular) carries both sensory and motor fibers. The motor component of $\mathrm{V} 3$ is responsible for muscle mastication, the tensor tympani, the tensor veli palatini, the mylohyoid, and the anterior belly of the digastric muscle. In Meckel's cave the three branches converge, with the cell bodies of incoming sensory nerve fibers located in a ganglion (the Gasserian or semilunar ganglion). The main roots of CNV and the Gasserian ganglion can be finely depicted by CISS in the prepontine cistern (see Figure 5) and in Meckel's cave, respectively. ${ }^{11}$ In cases of trigeminal neuralgia, CISS plays an important role in searching for vascular compressions. ${ }^{12}$

The Abducens Nerve

The abducens nerve (CNVI) innervates the lateral rectus muscle. It leaves the brainstem at the pontomedullary junction, then courses superiorly through the prepontine cistern and bends anteriorly to enter the Dorello's canal at the tip of the petrous apex. It is the only $\mathrm{CN}$ that is truly inside the cavernous sinus. The course of the abducens nerve and its relationship to the adjacent vessels can be reliably identified using CISS in the vast majority of cases (see Figure 6). ${ }^{13}$

The Lower Cranial Nerve Complex

The glossopharyngeal nerve (CNIX), the vagus nerve (CNX), and the cranial component of the spinal accessory nerve (CNXI) form the lower cranial nerve complex ( $\mathrm{LCNC}$ ). The nuclei of the LCNC are located in the medulla oblongata. These nerves arise from the medulla oblongata in the post-olivary sulcus as small rootlets in a linear array. ${ }^{14}$

\section{The Glossopharyngeal Nerve}

CNIX has both a cranial component and a spinal root, with a very complex physiology and numerous functions. It originates from the anterior and lateral portion of the medulla in the groove between the inferior olivary nucleus (ION) and the restiform body. It carries sensory information from the posterior third of the tongue, tonsils, pharynx and middle ear and supplies parasympathetic stimuli to the parotid gland. Additionally, it receives sensory fibers from the carotid bodies and supplies motor fibers to the stylopharyngeus muscle. CNIX passes anterior to the choroid plexus and cerebellar flocculus. It is the only nerve that enters the jugular foramen through the pars nervosa, which is a funnel-shaped dural meatus medial to the jugular spur (see Figure 7). The inferior petrosal sinus can be used as a landmark to identify CNIX, which is anterior to the glossopharyngeal meatus. ${ }^{14}$

\section{The Vagus Nerve}

CNX is the longest cranial nerve. It innervates the larynx, oesophagus, heart, lung, stomach, and intestines. On leaving the medulla between the ION and the inferior cerebellar peduncle, it extends through the pars vascularis of the jugular foramen, then passes into the carotid sheath to lie between the internal carotid artery and the internal jugular vein. The great majority of fibers composing CNX are afferent (sensory), conveying information from the viscera to the central nervous system. It supplies all of the intra-abdominal organs, with the exception of the 
adrenal glands and the distal second segment of the transverse colon. $\mathrm{CNX}$ is seen in its cisternal portion before entering the jugular foramen as one or two main roots (see Figure 8) at the level of the vagal trigone (a small bulge in the caudal portion of the floor of the fourth ventricle caused by the dorsal motor nucleus of CNX). ${ }^{14}$

\section{The Spinal Accessory Nerve}

CNXI has both a cranial and a spinal component. CNXI is purely motor, innervating the sternocleidomastoid and trapezius muscles. $\mathrm{CNXI}$ is not easily visualised, and in some situations coronal oblique reformats are necessary. However, root bundles that enter the pars vascularis but do not join the one or two main vagal roots can be classified as CNXI. The spinal roots of $\mathrm{CNXI}$ are those that rise through the foramen magnum and traverse the posterior fossa to reach the pars vascularis. ${ }^{14}$

\section{The Hypoglossal Nerve}

The hypoglossal nerve (CNXII) is a pure motor nerve that innervates the intrinsic and extrinsic tongue muscles (see Figure 9). The nucleus of CNXII is located medially to the dorsal nucleus of CNX and lies within the posterior and inferior aspects of the medulla oblongata. It lies close to the midline in the floor of the fourth ventricle, causing a focal bulge. CNXII exits the brainstem between the ION and the pyramid in the pre-olivary sulcus. The vertebral and posterior inferior cerebellar arteries are anterior and posterior to the cisternal portion of the CNXII, respectively. ${ }^{15}$

\section{Cysts and Cystic Lesions}

CISS is the sequence of choice to study cystic structures. Its application ranges broadly from the assessment of Virchow-Robin spaces (VRS) to the evaluation of cystic tumours such as dysembryoplastic neuroepithial tumours (DNET).

\section{Virchow-Robin spaces}

VRS are an extension of the subarachnoid space that surround the walls of arteries, arterioles, veins, and venules. The VRS signal should always follow that of the cerebrospinal fluid in all pulse sequences and should not enhance. Typically, VRS can occur in three locations: along the lenticulostriate arteries, inferior to the basal ganglia at the level of the anterior perforated substance (type 1); in the path of the perforating medullary arteries in the convexities, extending into the white matter (type 2) and in the midbrain (type 3) surrounding the collicular, accessory collicular, and thalamoperforating arteries, in the pontomesencephalic junction, and between the cerebral peduncles (see Figure 10). ${ }^{16}$

VRS are typically seen as well-defined oval, rounded, or tubular structures, depending on the plane in which they are imaged. They have regular margins, are commonly bilateral, and may be called enlarged when they measure more than $2 \mathrm{~mm} .{ }^{17}$ They can be atypical, presenting in clusters, or markedly large, causing mass effects, with odd shapes. They can even be misinterpreted as other pathologic processes, most often a cystic neoplasm. Although benign in some situations, dilated VRS can present with hydrocephalus due to compression of the cerebral aqueduct. ${ }^{16}$

\section{The Vestibular system}

CISS can play an important role in cases of sensorial hearing loss and also to study the vestibular system. It delivers fine detail when imaging the semi-circular canals and otolith organs (see Figure 11). ${ }^{17}$
Figure 9: Axial Constructive Interference in Steady State Image Showing the Oblique Course of the Hypoglossal Nerve (Large Arrow) as it Crosses the Lateral Perimedullary Cistern and Enters the Hypoglossal Canal (Small Arrow)

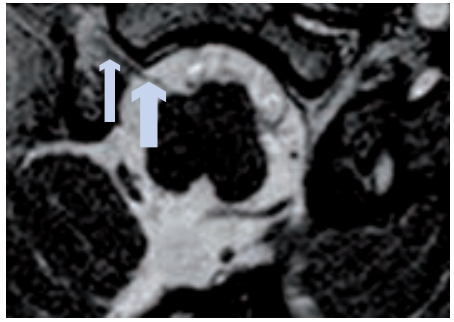

Figure 10: Axial Constructive Interference in Steady State Image at the Level of the Interpeduncular Fossa Showing the Usual 'Comet Tail' Appearance of a Dilated Perivascular Space in the Left Cerebral Peduncle

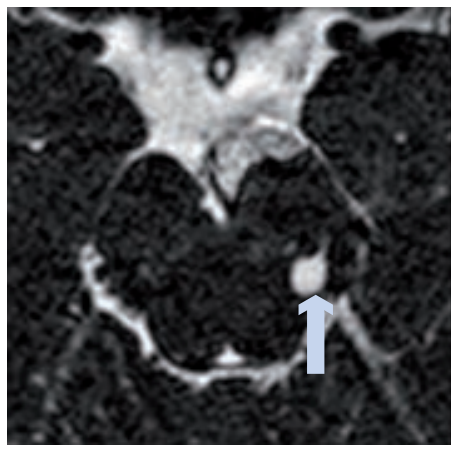

Figure 11: Maximum-intensity Projection of a Normal Membranous Labyrinth Using Constructive Interference in Steady State Images

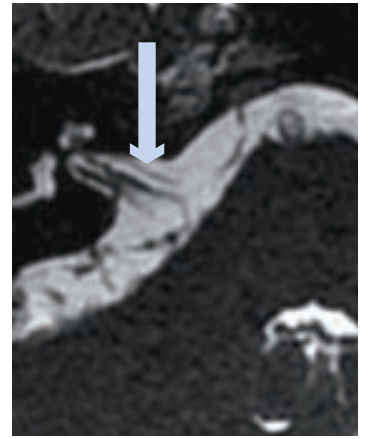

Figure 12: Sagittal 3D Constructive Interference in Steady State Reformatted Image at the Level of the Fourth Ventricle Showing an Intraventricular Cysticercus with a Scolex within It (Arrows)

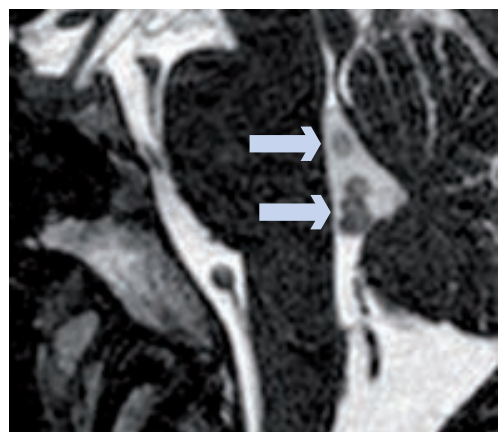




\section{Neurocysticercosis}

CISS is extremely useful for evaluating patients with neurocysticercosis (NCC), especially if the cestode is located in the ventricular system or subarachnoid space. CISS is the best single modality to depict the scolex inside the cysticercus cyst, particularly in the first stage of the disease when the cysticercus is antigenically non-viable and neither enhancement nor oedema is noted (see Figure 12).

In the second and third stages of NCC, the scolex may or may not be identified. In the fourth (involutional) stage, CISS is not adequate and other MRI sequences are more suitable for characterising the calcifying cystercus. ${ }^{18}$

\section{Hydrocephalus}

CISS is useful in patients with hydrocephalus. It is an excellent tool for assessing the cerebral aqueduct because it is capable of demonstrating minute membranes that may be seen in cases of noncommunicating hydrocephalus. ${ }^{19}$

\section{The Spine}

The use of CISS for spinal abnormalities is a more recent development. This sequence can be useful for characterising both intra- and extramedullary cystic abnormalities. ${ }^{20} \mathrm{CISS}$ can also be used to study the architecture of arteriovenous fistulas. ${ }^{21}$

\section{Conclusion}

In summary, CISS imaging is useful in the study of CNs, most notably when vascular compression tumours are suspected. CISS may also be of particular use in sensorineural hearing loss, for characterising the content of a cystic mass, and in cases of NCC. More recently, CISS has proved to be beneficial in spine imaging, especially in cases of intraspinal cystic lesions and arteriovenous malformations.
1. Chavhan GB, Babyn PS, Jankharia BG, et al., Steady-state MR imaging sequences: physics, classification, and clinical applications, Radiographics, 2008:28:1147-60.

2. Scheffler $\mathrm{K}$, Lehnhardt S, Principles and applications of balanced SSFP technique, Eur Radiol, 2003:13:2409-18.

3. Casselman JW, Kuhweide R, Deimling M, et al., Constructive interference in steady state-3DFT MR imaging of the inner ear and cerebellopontine angle, AJNR Am I Neuroradiol, 1993; 14:47-57

4. Girard N, Poncet M, Caces F, et al., Three-dimensional MRI of hemifacial spasm with surgical correlation, Neuroradiology, 1997;39:46-51.

5. Hermans R, Van der Goten A, De Foer B, Baert AL, MR screening for acoustic neuroma without gadolinium: value of 3DFT-CISS sequence, Neuroradiology, 1997:39:593-8.

6. Held P, Seitz J, Fründ R, et al., MRI detection of olfactory bulb and tract, I Neuroradiol, 2000;27:112-8.

7. Held P, Nitz W, Seitz J, et al., Comparison of $2 \mathrm{D}$ and $3 \mathrm{D}$ MRI of the optic and oculomotor nerve anatomy, Clin Imaging, 2000;24:337-43.

8. Liang C, Du Y, Lin X, et al., Anatomical features of the cisternal segment of the oculomotor nerve: neurovascular relationships and abnormal compression on magnetic resonance imaging, Neurosurg, 2009:111:1193-200.
9. Satoh T, Omi M, Ohsako C, et al., Visualization of aneurysmal contours and perianeurysmal environment with conventiona and transparent 3D MR cisternography. AINR Am I Neuroradiol ' 2005;26:313-8.

10. Yousry I, Morigg| B, Dieterich M, et al., MR anatomy of the proximal cisternal segment of the trochlear nerve: neurovascular relationships and landmarks, Radiology, 2002;223:31-8

11. Yousry I, Moriggl B, Schmid UD, et al., Trigeminal ganglion and its divisions: detailed anatomic MR imaging with contrast-enhanced 3D constructive interference in the steady state sequences, AJNR Am I Neuroradiol, 2005;26:1128-35

12. Yoshino N, Akimoto H, Yamada I, et al., Trigeminal neuralgia: evaluation of neuralgic manifestation and site of neurovascular compression with 3D CISS MR imaging and MR angiography, Radiology, 2003;228:539-45.

13. Yousry I, Camelio S, Wiesmann M, et al., Detailed magnetic resonance imaging anatomy of the cisternal segment of the abducent nerve: Dorello's canal and neurovascular relationships and landmarks, J Neurosurg, 1999;91:276-83.

14. Linn J, Moriggl B, Schwarz F, et al., Cisternal segments of the glossopharyngeal, vagus, and accessory nerves: detailed magnetic resonance imaging-demonstrated anatomy and neurovascular relationships, I Neurosurg, 2009;110:1026-41.

15. Yousry I, Morigg|B, Schmid UD, et al., Detailed anatomy of the intracranial segment of the hypoglossal nerve: neurovascular relationships and landmarks on magnetic resonance imaging sequences, I Neurosurg, 2002;96:1113-22.

16. Kwee RM, Kwee TC, Virchow-Robin spaces at MR imaging, Radiographics, 2007;27:1071-86.

17. Liang C, Zhang B, Wu L, et al., The superiority of 3D-CISS sequence in displaying the cisternal segment of facial, vestibulocochlear nerves and their abnormal changes, Eur J Radiol, 2010;74(3):437-40.

18. do Amaral $L$ L, Ferreira RM, da Rocha AJ, Ferreira NP, Neurocysticercosis: evaluation with advanced magnetic reurocysticercosis: evaluation with ad, Fenced magnetic resonance techniques and atypical forms, Top Magn Reson

19. Kurihara $\mathrm{N}$, Takahashi $\mathrm{S}$, Tamura $\mathrm{H}$, et al., Investigation of hydrocephalus with three-dimensional constructive interference in steady state MRI, Neuroradiology, 2000;42:634-8.

20. Gonçalves FG, Neves PO, Jovem CL, et al., Chronic myelopathy associated to intramedullary cysticercosis, spine (Phila Pa 1976), 2010;35:E159-62.

21. Ramli N, Cooper A, Jaspan T, High resolution CISS imaging of the spine, Br J Radiol, 2001;74:862-73. 


\section{TOUCHNEUROLOGY.COM}

\section{SUPPORTING THE NEUROLOGICAL COMMUNITY}

Touchneurology.com showcases articles from leading review journals European Neurological Review and US Neurology, alongside content from other highly respected institutions and authorities.

Through the dissemination of leading opinion and best practice, touchneurology.com aims to assist neurologists and healthcare administrators in continuously developing their knowledge, effectiveness and productivity.

- Contributions from leading voices in neurological medicine

- Timely comment and practice guidelines

- Comprehensive review and original research

- Event planners and symposium reviews

- Latest news and press releases

- Journal archive
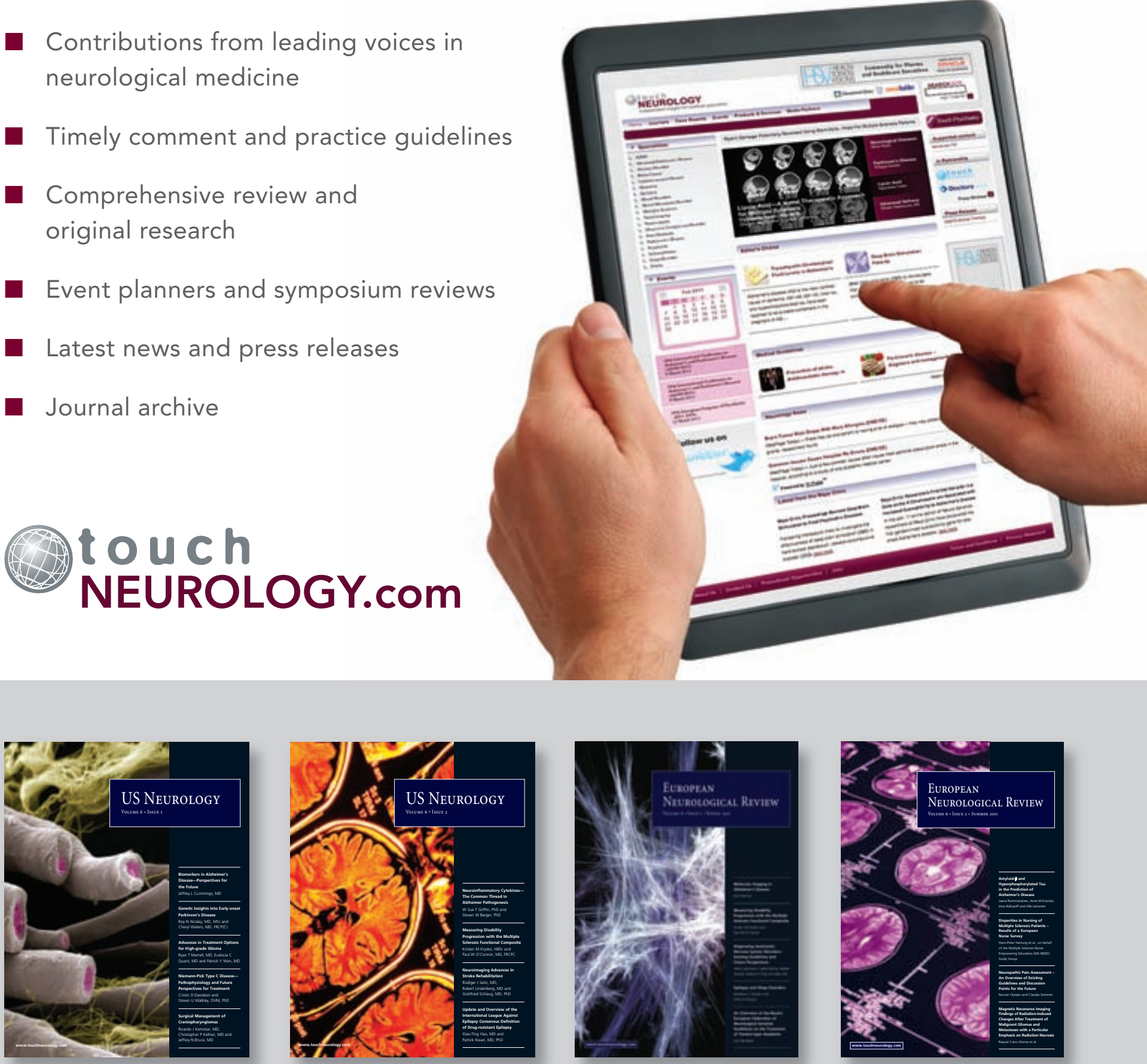


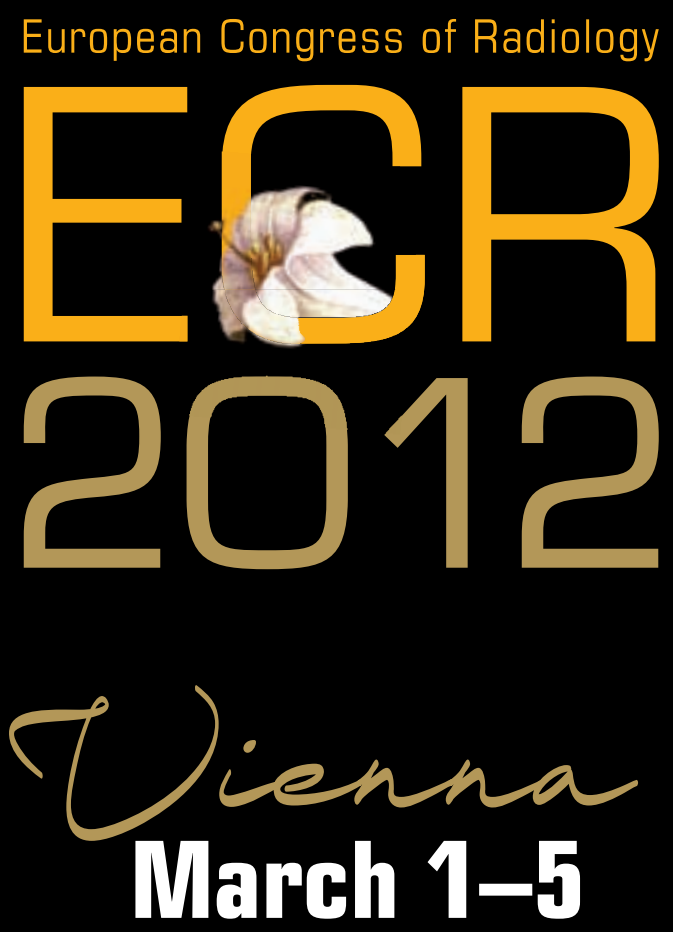

Abstract Submission Scientific Papers: July 5 - September 18, 2011 Poster Abstract Submission for EPOS ${ }^{\mathrm{TM}}$ : all year long! Online Registration: starts September 2011
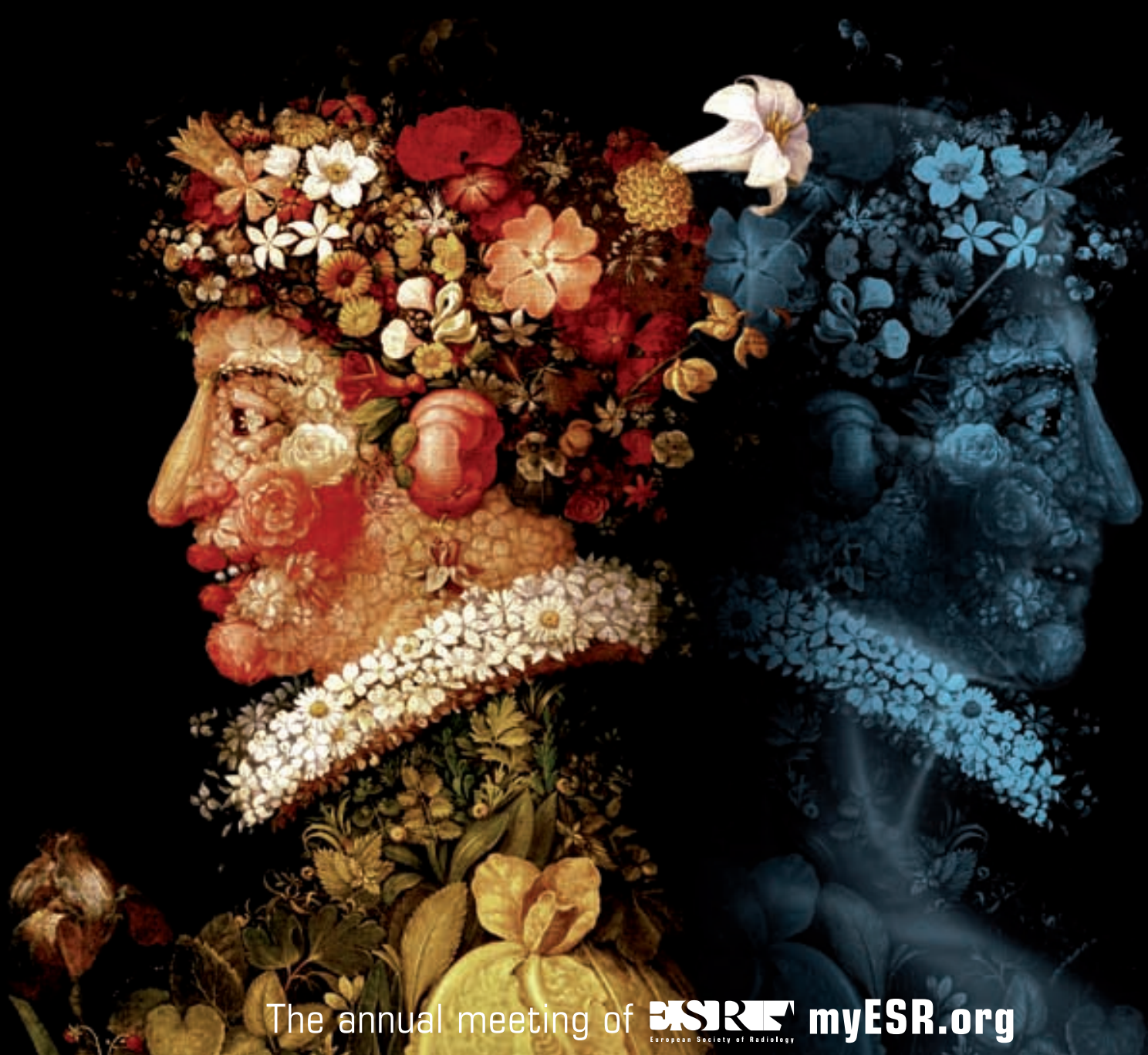
Twenty-second Meeting of the European Neurological Society

9-12 June 2012

\section{Prague, Czech Republic}

Neuralogy: Bearning, Bnowledge, progresis and the fulure

\section{Key symposia:}

4 Parkinson syndrome: an everlasting challenge

iㅜㄹ Neuroimaging and early diagnosis of neurological disease

4 Stem cells ready for clinical practice

4 MS and acquired demyelinating disorders

(4) Neurology and sleep-wake disorders

The congress programme includes 22 teaching courses, 11 workshops, practical sessions in clinical neurophysiology, interactive case presentations and selected scientific sessions in the form of oral and poster sessions.

Abstract submission deadline 26 January 2012 Early registration deadline: 22 March 2012

For further information please contact: ENS 2012, c/o Congrex Switzerland Ltd. Peter Merian-Strasse 80, 4002 Basel / Switzerland Phone +416168677 77 Fax +41616867788 Email basel@congrex.com

\section{www.ensinfo.org}




\section{SUPPORTING EXCELLELE III CLINICAL PRACTICE}

\section{w w w . t o u ch n e I r o I g g . c o m}

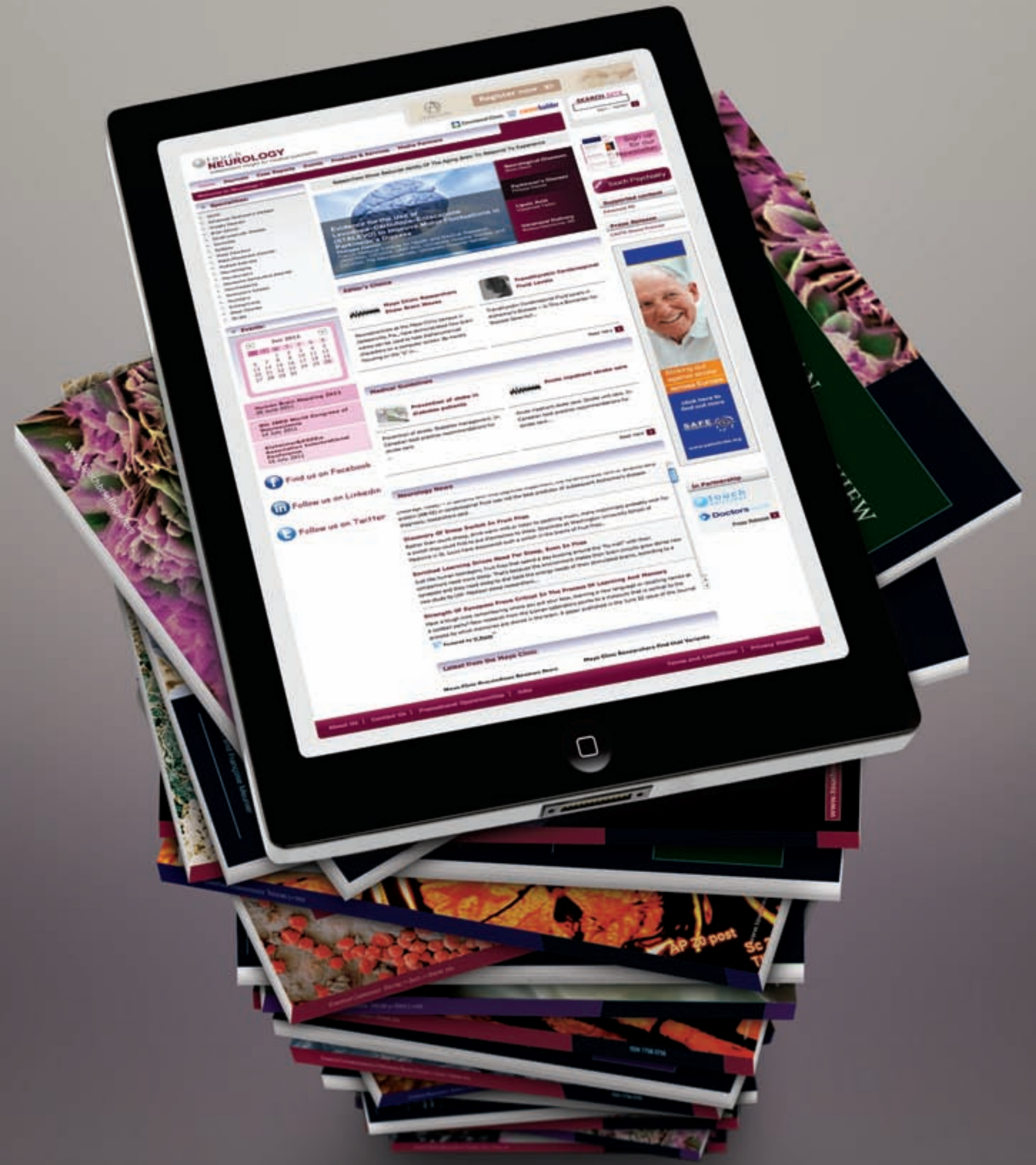

